\title{
n-Type Doping of Organic Semiconductors: Immobilization via Covalent Anchoring
}

Patrick Reiser, ${ }^{\S, \|,+}$ Frank S. Benneckendorf, ${ }^{\|, \perp, \ddagger}$ Marc-Michael Barf, ${ }^{\|, \#}$ Lars Müller, ${ }^{\|, \# \odot ~}$

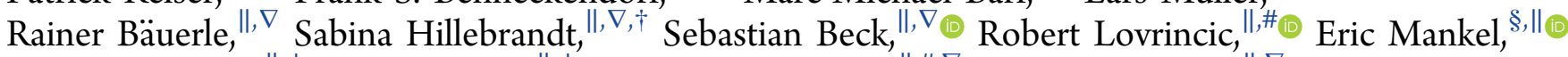
Jan Freudenberg, ${ }^{\|, \perp}$ Daniel Jänsch, ${ }^{\|, \perp}$ Wolfgang Kowalsky, ${ }^{\|, \#, \nabla}$ Annemarie Pucci, ${ }^{\|, \nabla}$, Wolfram Jaegermann, ${ }^{\S, \|}$ Uwe H. F. Bunz, ${ }^{\|, \perp_{(0)}}$ and Klaus Müllen*, ${ }^{*}, \bigcirc_{(1)}$

${ }^{\S}$ Materials Science Department, Surface Science Division, TU Darmstadt, Otto-Berndt-Straße 3, 64287 Darmstadt, Germany

"InnovationLab, Speyerer Straße 4, 69115 Heidelberg, Germany

${ }^{\perp}$ Organisch-Chemisches Institut, Ruprecht-Karls-Universität Heidelberg, Im Neuenheimer Feld 270, 69120 Heidelberg, Germany

\# Institute for High-Frequency Technology, TU Braunschweig, Schleinitzstraße 22, 38106 Braunschweig, Germany

${ }^{\nabla}$ Kirchhoff Institute for Physics, Heidelberg University, Im Neuenheimer Feld 227, 69120 Heidelberg, Germany

${ }^{\circ}$ Max Planck Institute for Polymer Research, Ackermannweg 10, 55128 Mainz, Germany

Supporting Information

ABSTRACT: Electrical doping is an important tool in the design of organic devices to modify charge carrier concentration in and Fermi level position of organic layers. The undesired diffusion of dopant molecules within common transport materials adversely affects both lifetime and device performance. To overcome this drawback, we developed a strategy to achieve immobilization of dopants through their covalent attachment to the semiconductor host molecules. Derivatization of the commonly employed n-type dopant 2-(2-methoxyphenyl)-1,3-dimethyl-2,3-dihydro-1H-benzoimidazole (o-MeO-DMBI) with a phenylazide enables the resulting $\boldsymbol{o}$-AzBnO-DMBI to photochemically generate a reactive nitrene, which subsequently binds covalently to the host material, 6,6-phenyl- $\mathrm{C}_{61}$-butyric acid methyl ester (PCBM). Both the activation and addition reactions are monitored by mass spectrometry as well as optical and photoelectron spectroscopy. A suppression of desorption and a decrease in volatility of the DMBI derivative in ultrahigh vacuum were observed after activation of a bilayer structure of PCBM and $\boldsymbol{o}$-AzBnO-DMBI. Electrical measurements demonstrate that the immobilized o-AzBnO-DMBI can (i) dope the PCBM at conductivities comparable to values reported for $\boldsymbol{o}-\mathbf{M e O}-\mathbf{D M B I}$ in the literature and (ii) yield improved electrical stability measured in a lateral two terminal device geometry. Our immobilization strategy is not limited to the specific system presented herein but should also be applicable to other organic semiconductor-dopant combinations.

\section{INTRODUCTION}

The applications and benefits of electrical or electrochemical doping in organic semiconductors are manifold. ${ }^{1,2}$ Suitable dopants increase the conductivity of organic layers by several orders of magnitude, for example, in charge transport layers of organic light-emitting diodes (OLEDs). ${ }^{3}$ Promising thermoelectric devices can be realized with doped conjugated polymers due to their low thermal conductivity. ${ }^{4-6}$ Most importantly, doped layers enable quasi-ohmic contacts to metal electrodes, which greatly improves charge carrier injection and mitigates contact resistances due to offsets in energy level alignment. $^{7-9}$ A prerequisite for applying doped layers in dedicated device stacks is stability since diffusion of dopants into adjacent active layers has been identified as a degradation mechanism (e.g., exciton quenching), detrimental to photoactive devices. $^{10-14}$ Moreover, diffusion of dopants can be problematic in organic hole transport layers for planar perovskite solar cells. ${ }^{15}$ Consequently, stable and efficient doping of organic materials is of high interest in present-day device fabrication. Replacing atomic dopants such as halides or alkali metals by small molecular p- and n-type dopants reduces volatility significantly. ${ }^{16}$ However, some host-dopant systems still exhibit diffusivity of organic electron donors/acceptors. $^{17,18}$ This effect is more pronounced within conjugated polymers compared to molecular host materials. ${ }^{19-21}$ Even though the dopant lacks diffusion in the host matrix, this might change when an electric field is applied. Recently, the migration of molecular dopants in an electric field has been

Received: March 22, 2019

Revised: $\quad$ May 14, 2019

Published: May 15, 2019 
reported in poly(3-hexylthiophene) (P3HT) and is considered as a drift effect. ${ }^{22}$ To suppress dopant diffusion and drift, certain strategies have been proposed and demonstrated, for example, introduction of a dense dopant blocking interlayer or filling of dopant pathways using a second redox-inactive transport material. ${ }^{23-25}$ However, processing becomes more difficult, and possible complications concerning charge transport or energy level alignment arise from the additional dopant blocking materials. ${ }^{26}$ Furthermore, polar side chains were introduced to a polymer to increase its dopant affinity, yielding an improved thermal stability of molecular dopants and a higher melting temperature. ${ }^{25}$ Larger molecular dopants offer a better morphological stability as demonstrated by thermally activated diffusion of dopants in molecular host systems. ${ }^{27}$ Besides physical modifications such as size or morphology, increased intermolecular interaction also reduces the mobility of dopants. These include dispersion force, dipole-dipole interactions, $\pi-\pi$ interactions, or even hybridization. ${ }^{28}$ Charged dopants are much less diffusive than neutral dopants due to electrostatic interactions. ${ }^{21,29}$ A covalent bond between the dopant and the host molecule would allow much stronger "fixation" ideally for the immobilization of both neutral and charged dopants. A premodification of the host with a functional group that introduces self-doping was already used to localize dopants in assembled thin films. ${ }^{30,31}$ For the covalent bond formation after blending, the host and dopant have to have complementary functional groups. ${ }^{32-34}$ This concept requires modification of each transport material to fit the corresponding dopant, which is not practical given the plethora of materials in use. In order to be generally compatible with a wide scope of host materials, an anchor group for dopants needs an unspecific binding method, for example, by attachment to aliphatic $\mathrm{C}-\mathrm{H}$ or olefinic $\mathrm{C}=\mathrm{C}$ bonds present in almost any organic semiconductor without introduction of severe degradation or traps. A practical solution for this issue can be derived from photochemical cross-linking as the desolubilization protocol: ${ }^{35}$ The utilization of various bis-azides for cross-linking, thus preventing polymer strands from redissolution in solution-processed heterostructures, is not limited to one specific polymer. ${ }^{36,37}$ The azide is activated by deep-ultraviolet light (UV, $254 \mathrm{~nm}$ ), inducing photolysis and generating reactive singlet nitrenes. When properly substituted, these predominantly insert into aliphatic $\mathrm{CH}$ bonds, and side reactions ${ }^{38}$ involving triplet pathways, aromatic insertion, attack upon other functional groups, or ketenimine ring expansion are suppressed. ${ }^{36,39,40}$ The latter is found to be dominating only in solution for non-fluorinated aryl azides, ${ }^{39,41}$ whereas in thin films or matrices, $\mathrm{CH}$ insertion prevails. $^{42-45}$

1,3-Dimethyl-2-phenyl-2,3-dihydro- $1 H$-benzoimidazole (DMBI) derivatives are effective, air-stable, and thus easy-tohandle, solution-processable n-type dopants. DMBIs are often used to dope n-channel semiconductors, preferably PCBM $^{46-51}$ or n-type polymers. ${ }^{47,52}$ However, they seem to be more effective, in terms of achieved maximum conductivity, in combination with PCBM than with polymers like $\mathrm{P}$ (NDI2OD-T2) due to the poor miscibility of the dopant and polymer. ${ }^{6,47,53}$ Although the doping mechanism of DMBI derivatives in, for example, PCBM has been investigated, it is still under debate. ${ }^{48,51,54}$ The initial step either involves a hydride transfer to the host or a hydrogen radical transfer with a subsequent electron transfer-both mechanisms lead to the generation of cationic DMBIs and PCBM radical anions responsible for the doping effect.

In this work, we report the successful anchoring of an azidefunctionalized DMBI-based dopant in PCBM, employing a nonspecific immobilization strategy through covalent attachment to the host while retaining its dopant strength compared to a nonfunctionalized counterpart. We detail the synthesis of $o$-AzBnO-DMBI and o-BnO-DMBI, in which the orthomethoxy group of $\boldsymbol{o}$-MeO-DMBI ${ }^{5,56}$ is substituted by a (4azidobenzyl)oxy and a benzyloxy functional group, respectively, and investigate their doping and anchoring properties in blends with PCBM. Infrared and photoelectron spectroscopies are employed to analyze the $\mathrm{CH}$ insertion or cycloaddition reaction, and conductivity measurement was employed to verify doping with and without a UV trigger. High-resolution mass spectrometry verifies a covalent linkage of the dopant to PCBM. Our immobilization strategy via nitrene generation is generally applicable and not limited to a specific host-dopant system. Furthermore, it is destined to improve the stability, lifetime, and performance of doped organic devices by ideally suppressing both detrimental diffusion and drift effects.

\section{RESULTS AND DISCUSSION}

Synthesis and Immobilization Reactions. DMBI dopants are easily accessible via simple condensation reactions according to Scheme 1. Introduction of a phenylazide is

Scheme 1. Synthesis of $o$-AzBnO-DMBI and $o$-BnO-DMBI
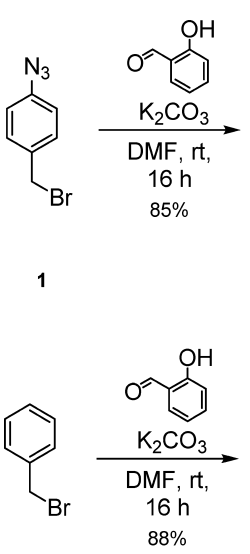

3

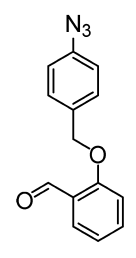

2

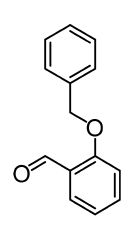

4
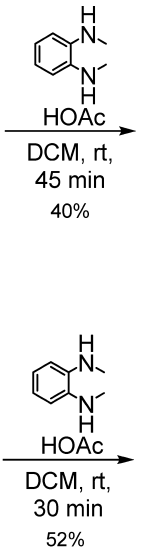

$52 \%$

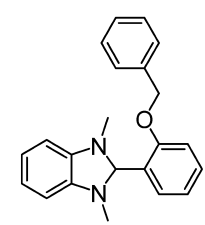

o-BnO-DMBI achieved after etherification of salicylaldehyde employing 1azido-4-(bromomethyl)benzene (1) in DMF at room temperature furnishing compound 2 in $85 \%$ yield, followed by condensation with $N^{1}, N^{2}$-dimethylbenzene-1,2-diamine to afford o-AzBnO-DMBI in $40 \%$ yield. The characteristic IR absorption band of the azide stretching vibration at $2109 \mathrm{~cm}^{-1}$ (see Figure $4 \mathrm{~b}$ and Figure S11) confirms the azide functionalization. ${ }^{57}$

To elucidate the influence of the azide group on immobilization, we synthesized the non-azido-functionalized analogue and thus nonimmobilizable $\boldsymbol{o}$-BnO-DMBI employing benzyl bromide (3) for the Williamson ether synthesis. Both new dopants were isolated after crystallization from petroleum ether/methanol or DCM/hexane, respectively. The obtained colorless single crystals were suitable for X-ray crystal analyses, unambiguously proving their structure (see the Supporting Information). In order to optimize the processing parameters, 
a) DMBI charge transfer

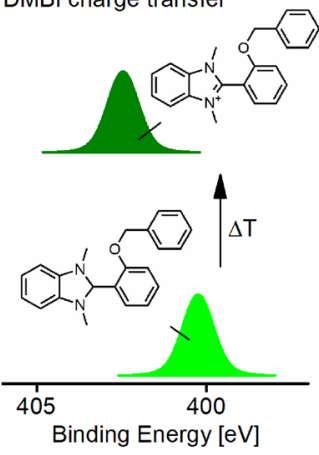

d)

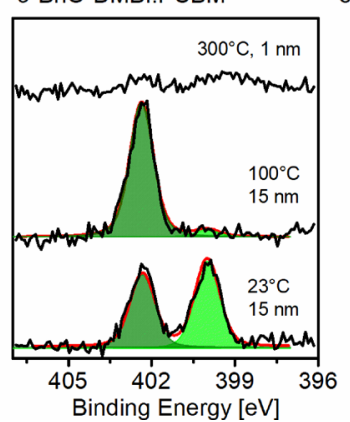

b)

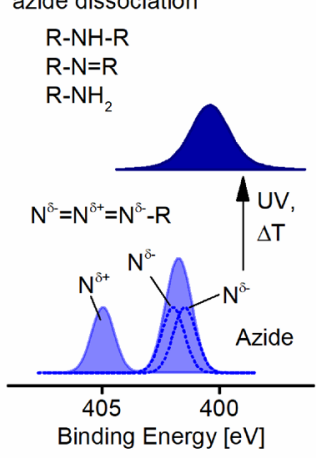

c) Idealized $\mathrm{N}$ 1s for o-AzBnO-DMB

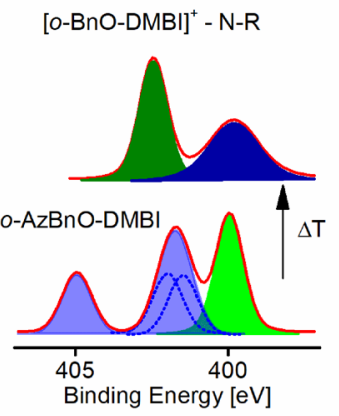

e) o-AzBnO-DMBI:PCBM + UV

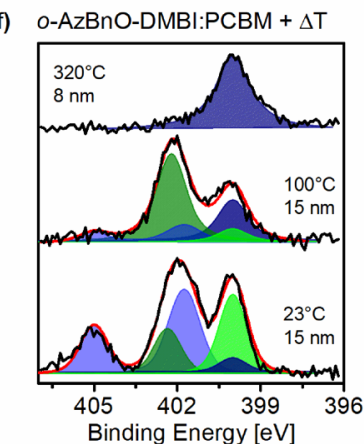

Figure 1. XPS measurements of the $\mathrm{N}$ 1s core-level spectra of thermally annealed PCBM films doped with o-BnO-DMBI and $\boldsymbol{o}$-AzBnO-DMBI in panels $(\mathrm{d}-\mathrm{f})$. $(\mathrm{a}-\mathrm{c})$ Schematic cartoon of $\mathrm{N}$ 1s peak components and their transformation. (d) Recorded spectra of PCBM doped with $o$-BnODMBI for different annealing temperatures. (e) N 1s signal of PCBM doped with $\boldsymbol{o}$-AzBnO-DMBI together with the spectrum measured after UV activation and after annealing of the UV-activated film to $320^{\circ} \mathrm{C}$. (f) Direct annealing of the o-AzBnO-DMBI/PCBM film without UV treatment. The assignment of peak composition in panels $(\mathrm{d}-\mathrm{f})$ is inferred from contributions of DMBI in green and from the azide in blue. The expected peak components of DMBI upon charge transfer and decomposition of pure azide are sketched in panels (a) and (b), respectively. For o-AzBnODMBI, both reactions of panels (a) and (b) can occur as illustrated in panel (c). The peak shapes of panels (a) and (b) are fitted to the data in panels $(e-f)$ using a constrained model (see the Supporting Information for details). In the component fit, there is already a considerable fraction of charged dopants in panels $(\mathrm{d}-\mathrm{f})$ and possibly dissociated azide in panels $(e)$ and $(\mathrm{f})$ at room temperature. The initial film thickness at room temperature is $15 \mathrm{~nm}$ and changes in panels $(\mathrm{d}-\mathrm{f})$ for higher temperatures.

we studied the stability of the newly synthesized dopants and the literature known $\boldsymbol{o}$-MeO-DMBI in solution (see the Supporting Information, Figure S13). NMR spectroscopy revealed that the dopants are only fairly stable in solution: after 1 day at ambient conditions (sunlight and air) in chloroform, oxidized species can be seen. However, the dopant appears to be stable for processing within several hours. In the solid state at $-10{ }^{\circ} \mathrm{C}$, however, no degradation was observed over months.

In acetonitrile, the UV-vis spectrum of $\boldsymbol{o}$-BnO-DMBI exhibits absorption features at $\lambda=221,274$, and $313 \mathrm{~nm}$, whereas for $\boldsymbol{o}$-AzBnO-DMBI, an additional absorption band at $253 \mathrm{~nm}$ is detected and attributed to the azide functional group (Figure S14). ${ }^{42,58}$ Irradiation at $254 \mathrm{~nm}$ excites the azide and induces nitrene generation. ${ }^{42,45}$ Throughout this work, this wavelength is used to initiate the immobilization process. Thermogravimetric analysis (see Figure S15) of o-AzBnODMBI shows irreversible mass loss at $117^{\circ} \mathrm{C}$ corresponding to the extrusion of nitrogen. During additional heating/cooling cycles below the decomposition temperature of $240{ }^{\circ} \mathrm{C}$, no further mass loss is observed. Similar to the azide-functionalized dopant, $\boldsymbol{o}$-BnO-DMBI exhibits a rather low melting point at $108{ }^{\circ} \mathrm{C}\left(86^{\circ} \mathrm{C}\right.$ for $\boldsymbol{o}$-AzBnO-DMBI $)$ and decomposes at $200{ }^{\circ} \mathrm{C}$. To achieve maximum doping effects for the host/ DMBI blend, annealing at $75{ }^{\circ} \mathrm{C}$ is required. ${ }^{48}$ Thus, oAzBnO-DMBI is stable enough to test the doping of the PCBM host upon thermal treatment.
In the following, we provide spectroscopic data to corroborate successful anchoring of the o-AzBnO-DMBI dopant. From X-ray photoelectron spectroscopy (XPS) corelevel spectra, we gain insight into the activation mechanism of both the dopant and the anchor group as well as possible decomposition reactions. In order to additionally test the thermal stability of the DMBI derivative in the blend, we annealed thin films of high doping concentration (50 mol \%) in ultrahigh vacuum (UHV, $10^{-9} \mathrm{mbar}$ ) and measured XPS at each temperature step (for details, see Figure S20). The N 1s emission of $\boldsymbol{o}$-AzBnO-DMBI contains a contribution of the functional azide group and the DMBI core, illustrated in Figure $1 \mathrm{c}$ in blue (azide) and in green (DMBI).

To assign the $\mathrm{N}$ 1s peak components of $\boldsymbol{o}$-AzBnO-DMBI correctly, it is instructive to analyze spectral changes expected from DMBI and the azide group, sketched in Figures 1a and $1 \mathrm{~b}$, respectively. The spectra of $\boldsymbol{o}$-BnO-DMBI in Figure 1d only exhibit peak components of the DMBI core located at 402.5 and $400 \mathrm{eV}$. We identify the nitrogen emission at 402.5 $\mathrm{eV}$ as charged dopants having formed a radical and transferred an electron to PCBM. This assignment agrees with the $\mathrm{N}$ 1s emission of the ionic DMBI salt at about $402.5 \mathrm{eV}$ reported in the literature. ${ }^{59}$ With rising temperature, the peak component at $400 \mathrm{eV}$ transforms into charged DMBI species at $402.5 \mathrm{eV}$, which correlates with an increase in conductivity observed for annealed PCBM films doped with DMBI. The $\mathrm{N}$ 1s signal reported for pure azides sketched in Figure $1 \mathrm{~b}$ has three 
components, whereas two components coincide between 400 and $402 \mathrm{eV}$ leaving a single peak component isolated at high binding energies. ${ }^{60,61}$ From density functional theory (DFT) calculations, it is known that the central nitrogen in $\mathrm{R}-\mathrm{N}=$ $\mathrm{N}^{\delta+}=\mathrm{N}^{\delta-}$ is electron-deficient and has a $\mathrm{N} 1 \mathrm{~s}$ level at high binding energies. ${ }^{62}$ Azide decomposition triggered by UV light or thermal energy leads to nitrogen release and ideally to the generation of singlet nitrene, which can then form new carbon-nitrogen bonds. Regarding the azide in o-AzBnODMBI, the peak component at $405 \mathrm{eV}$ in Figure 1e was therefore assigned to the central nitrogen, and the contributions of the lateral nitrogen atoms were between 400 and 402 $\mathrm{eV}$. Upon UV activation at room temperature in Figure 1e, the former peak at $405 \mathrm{eV}$ vanishes completely, reducing the peak area around $402 \mathrm{eV}$ and increasing at $400 \mathrm{eV}$. During annealing of the doped PCBM film in Figure 1f, the N 1s emission at 405 $\mathrm{eV}$ disappears above $100{ }^{\circ} \mathrm{C}$ suggesting that the azide group becomes thermally activated and produces a nitrene. As a result, carbon-nitrogen bonds are formed causing an additional $\mathrm{N} 1 \mathrm{~s}$ emission at $400 \mathrm{eV}$, which persists at temperatures higher than $300{ }^{\circ} \mathrm{C}$. At the same time, the DMBI part of $\boldsymbol{o}$ AzBnO-DMBI donates an electron, similar to o-BnO-DMBI, thus transferring the peak component at $400 \mathrm{eV}$ into the charged DMBI species at $402.5 \mathrm{eV}$. When reaching higher temperature in UHV, pure and o-BnO-DMBI-doped PCBM completely evaporate from the substrate at 300 and $200{ }^{\circ} \mathrm{C}$, respectively. Interestingly, the film doped with o-AzBnODMBI is stable even beyond $300{ }^{\circ} \mathrm{C}$, and only a partial decrease in coverage is observed. However, from the $\mathrm{N}$ 1s emission, we infer that the $\mathrm{N} 1$ s peak component at $402.5 \mathrm{eV}$, attributed to charged dopants, disappears almost completely above $210{ }^{\circ} \mathrm{C}$ leaving only carbon-nitrogen bonds at $400 \mathrm{eV}$ when approaching $320^{\circ} \mathrm{C}$ in Figure 1 (for details, see Figure S20).

At room temperature, pure and untreated o-AzBnO-DMBI is volatile under UHV conditions as observed by XPS. However, when o-AzBnO-DMBI is sequentially processed on top of PCBM in monolayer-range thickness, a desorption experiment in $\mathrm{UVH}$ at room temperature reveals that the anchoring of o-AzBnO-DMBI also appears to be quantitative, rendering $\boldsymbol{o}$-AzBnO-DMBI completely resistant to desorption after UV activation (see the Supporting Information for details). A similar trend is observed for thicker bilayer structures, which means that in pure dopant films o-AzBnODMBI may also react with neighboring dopants (see Figure S19).

Concerning the anchoring mechanism, it is known that azides react with fullerenes in a 1,3-dipolar $[3+2]$ cycloadditions affording triazolines, ${ }^{63,64}$ which, upon thermal treatment, undergo loss of nitrogen, ${ }^{65,66}$ or $[2+1]$ cycloaddition with nitrenes yielding aziridinofullerenes or azafulleroids. ${ }^{67,68}$ Additionally, $\mathrm{CH}$ insertion into the alkyl chains of PCBM, though much less probable, may occur. To demonstrate covalent attachment of the dopant to the host, blends of $\boldsymbol{o}$-AzBnO-DMBI and PCBM were analyzed by electrospray ionization (ESI) and matrix-assisted laser desorption ionization (MALDI) high-resolution mass spectrometry. We prepared blends of PCBM and o-AzBnO-DMBI at $25 \mathrm{~mol} \%$ in a solution of chlorobenzene, which were subsequently dried in vacuo. Azide activation was achieved by either annealing at $135^{\circ} \mathrm{C}$ or UV activation of spin-cast thin films, which were redissolved, and the material was dried afterward. Whereas no $[3+2]$ cycloaddition products are found after simple blending, additional peaks of covalent PCBM-nitrene adducts at $1253.2 \mathrm{~m} / z$ (attributed to [PCBM + $\left.\left(\text { o-AzBnO-DMBI)- } \mathrm{H}-\mathrm{N}_{2}\right]^{+}\right)$are detected after heating to 135 ${ }^{\circ} \mathrm{C}$ (thermal azide generation) or photochemical activation (Figure 2 and Figures S24 and S26). Photochemical activation

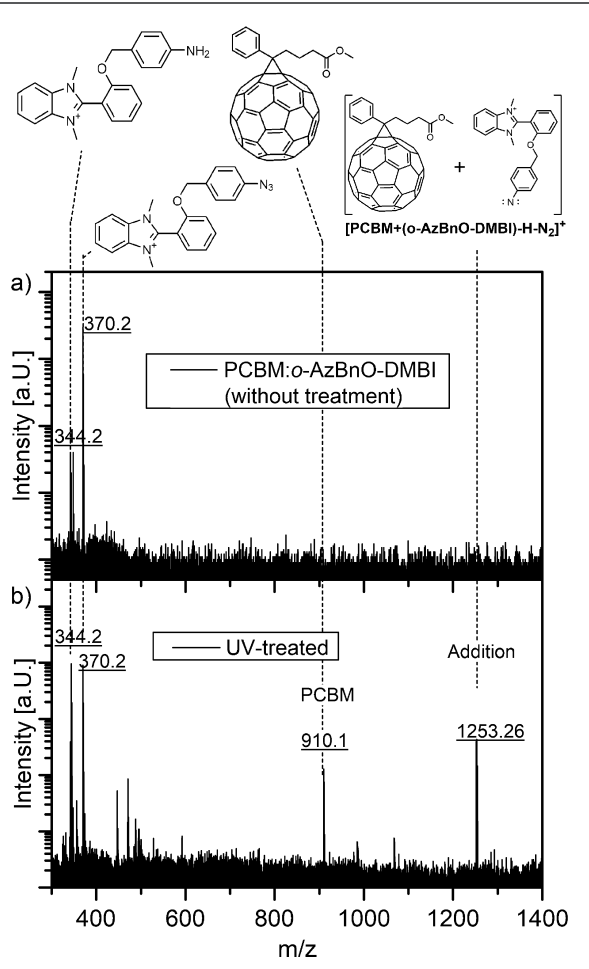

Figure 2. MALDI high-resolution mass spectrometry data are plotted for (a) a blend of PCBM and o-AzBnO-DMBI and (b) after UV treatment on a log scale. In principle, the MALDI laser used for desorption can cause azide decomposition, but we find a pronounced main peak at $370.2 \mathrm{~m} / z$ assigned to intact $\boldsymbol{o}$-AzBnO-DMBI in panel (a). Comparable ESI spectra can be found in Figures S24-S26. Only trace amounts of unfavorable amine products are detected at $344.2 \mathrm{~m} /$ $z$ in both panels (a) and (b) relative to $\boldsymbol{o}$-AzBnO-DMBI at $370.2 \mathrm{~m} / z$.

is the more benign method as shown in the ESI spectra (Figure S25); fragmentation of the ether bond within the thermal activated blends is detected. We find comparably small mass peaks for amines (these may also be a fragment of mass spectroscopy created during ionization $)^{69,70}$ and unreacted azide from the UV-activated blends (Figure 2). We attribute the latter to the slightly modified sample preparation for mass spectroscopy because XPS and IR experiments (Figures 3 and 4, respectively) demonstrate quantitative azide photolysis.

Doping and Functional Characterization. The conductivity measurements were performed under nitrogen on pure and doped PCBM layers (host-to-dopant ratio, 10:1), spin-cast from chlorobenzene onto prestructured silver contacts on glass substrates (Figure $3 \mathrm{a}$ and Figure S17). Whereas neat PCBM thin films exhibit conductivities in the range of $10^{-6} \mathrm{~S} / \mathrm{m}$, this characteristic value rises up to 0.1 and $0.01 \mathrm{~S} / \mathrm{m}$ for as-cast films doped with $\boldsymbol{o}$-AzBnO-DMBI and $\boldsymbol{o}$ BnO-DMBI, respectively. The final annealing step for doped films at $75{ }^{\circ} \mathrm{C}$ increases the conductivity further to $1 \mathrm{~S} / \mathrm{m}$. After UV treatment $(254 \mathrm{~nm})$ of as-cast films, the conductivities of the doped PCBM layers decrease by roughly half an order of magnitude in comparison to as-cast blends. The subsequent thermal treatment $\left(1 \mathrm{~h}\right.$ at $\left.75^{\circ} \mathrm{C}\right)$ increases the 

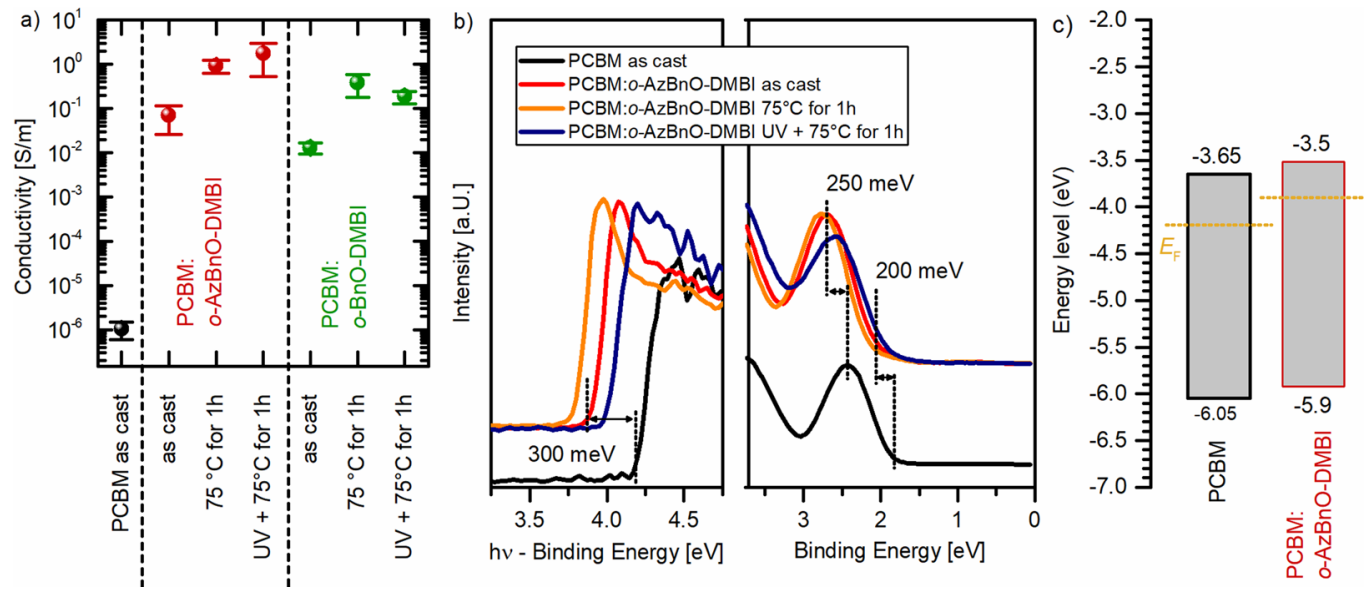

Figure 3. (a) Conductivities of pure and doped PCBM layers with a molar doping ratio of 10:1 as-cast, after UV treatment, and after subsequent annealing. (b) UPS spectra of pure and doped PCBM layers with a molar doping ratio of 10:1 as-cast, after UV treatment, and after subsequent annealing. The secondary electron cutoff with respect to the vacuum level and the HOMO onset with respect to the Fermi level are given. (c) Calculated energy levels of pure and doped PCBM layers with a band gap of $2.4 \mathrm{eV}$.
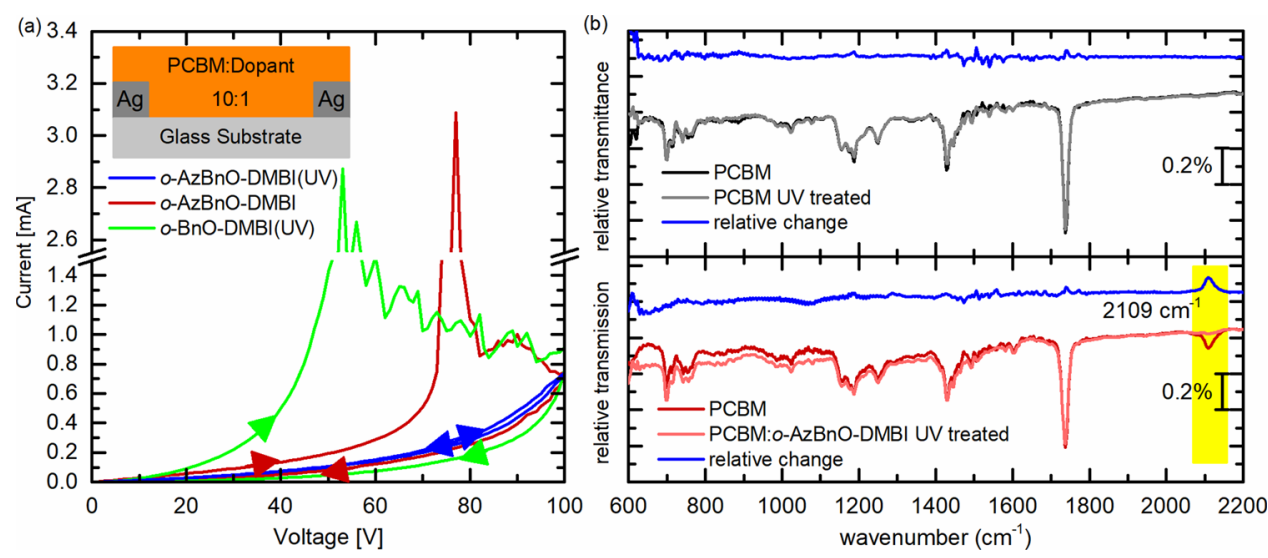

Figure 4. (a) $I-V$ curves of annealed layers as-cast and after UV treatment. (b) Relative IR transmission spectra of $30 \mathrm{~nm}$ thick pure and doped PCBM layers with a molar doping ratio of 10:1. The spectra were measured on as-cast layers and after UV treatment as given in the legend. Dividing the spectra after the UV treatment by the as-cast spectra reveal the relative spectral change caused by the UV treatment. The vibrational mode of the azide group at $2109 \mathrm{~cm}^{-1}$ is marked in panel (b) and strongly reduced by the UV treatment.

conductivities for both dopants by more than 2 orders of magnitude to $1 \mathrm{~S} / \mathrm{m}$. In total, this is a gain of 6 orders of magnitude compared to neat PCBM layers (see Figure 3a).

UV photoelectron spectroscopic (UPS) measurements of corresponding PCBM layers on silicon are presented in Figure 3b. The secondary electron cutoffs of o-AzBnO-DMBI-doped PCBM reveal strong shifts, which translate to a reduction of the work function of about $300 \mathrm{meV}$ compared to the neat PCBM layer. Additionally, in doped layers, the highest occupied molecular orbital (HOMO) broadens, and the HOMO maximum shifts by $250 \mathrm{meV}$ to higher binding energies compared to undoped PCBM and is accompanied by a shift of the core-level emission lines (see Figure S19). For comparison, Naab et al. observed a Fermi level shift of 500 $\mathrm{meV}$ in a PCBM blend with 1 wt \% o-MeO-DMBI on ITO. ${ }^{51}$

The Fermi level shift goes along with an increase of free charge carrier concentration and thus leads to an increased conductivity revealing a strong n-type doping effect of both DMBI derivatives in PCBM. Annealing of the thin film results in an additional shift of the Fermi level toward the LUMO of about 50-100 meV for o-AzBnO-DMBI arriving at a total $350-400 \mathrm{meV}$ shift. Furthermore, we find a broadening of the emission of the HOMO level, yielding an increase of tail states for doped PCBM films. The results of the UPS measurements were used to calculate the energy level diagrams of pure and doped PCBM layers that are depicted in Figure 3c, assuming a constant band gap of about $2-2.5 \mathrm{eV} .^{71-73}$

Figure 4a shows $I-V$ curves of doped o-AzBnO-DMBI and $\boldsymbol{o}$-BnO-DMBI PCBM layers as-cast and after UV treatment. At high electric field strength, the untreated films including $o$ AzBnO-DMBI feature a pronounced rise and subsequent falloff in current. As a result, the $I-V$ curve displays a distinct hysteresis, which can be also observed when repeating the cycle (slightly shifted to higher voltages). This can be explained either by contact modification and/or by ionic migration. A drift of charged dopant molecules away from a contact causes a similar hysteresis in $I-V$ curves, which was found for films of poly(3-hexylthiophen-2,5-diyl) (P3HT) doped with the p-dopants 2,3,5,6-tetrafluoro-7,7,8,8-tetracyanoquinodimethane (F4TCNQ) and molybdenum tris[1(methoxycarbonyl)-2-(trifluoromethyl)-ethane-1,2-dithiolene ] $\left.\left(\mathrm{Mo}\left(\mathrm{tfd}-\mathrm{CO}_{2} \mathrm{Me}\right)_{3}\right)\right){ }^{22}$ On the other hand, the $I-V$ curve of an o-AzBnO-DMBI doped layer that was UV-treated (followed by a final annealing step) displays no or reduced hysteresis on average. This observation is a first indication for a successful dopant immobilization after nitrene generation. 
Moreover, hysteresis persists in $\boldsymbol{o}$-BnO-DMBI-doped and UVtreated PCBM, suggesting that the observed characteristic is not due to changes in morphology during UV irradiation but due to the reactions of $\boldsymbol{o}$-AzBnO-DMBI.

The HOMO level of PCBM broadens after UV exposure, which is attributed to an oxidation of the thin film surface most likely from residual oxygen in the glovebox triggered by UV light (measured by XPS in Figure S18). IR transmission measurements of PCBM layers before and after UV exposure, given in Figure $4 b$, do not show an oxidation of the PCBM bulk material, meaning the observed oxidation takes place only at the surface. Unfortunately, this hinders the further evaluation of the UV-treated doped PCBM layers by UPS since the observed surface degradation strongly influences the measured Fermi level position. IR transmission measurements of PCBM layers doped with o-AzBnO-DMBI before and after $\mathrm{UV}$ treatment are presented in Figure $4 \mathrm{~b}$ (see the Supporting Information for additional spectra). Due to the doping concentration of $10 \mathrm{~mol} \%$, most of the observed vibrational modes stem from PCBM and fail to reveal a change caused by the UV treatment. Only the characteristic vibrational mode of the dopant's azide group at $2109 \mathrm{~cm}^{-1}$ vanishes completely, revealing a quantitative activation and dissociation of the azide by UV light.

\section{CONCLUSIONS}

In this work, we detail the synthesis of o-AzBnO-DMBI, a derivative of the literature known o-MeO-DMBI. An azidophenyl group is introduced to DMBI by etherification of salicylaldehyde employing 1-azido-4-(bromomethyl)benzene and a subsequent condensation with $N^{1}, N^{2}$ dimethylbenzene-1,2-diamine. The modification of DMBI with a reactive azide aims at providing a universally applicable anchor-like functional group, which should be able to covalently bind to a wide scope of common organic semiconductors. By creating a covalent bond with the host material, the dopant is expected to be immobilized or at least rendered significantly less mobile, which ideally reduces problems in devices arising from diffusion or drift of dopants. In an exemplary study, o-AzBnO-DMBI was blended with PCBM and activated by UV light in order to generate highly reactive nitrenes to ultimately form covalent carbon-nitrogen bonds between PCBM and the dopant. The azide activation was monitored by infrared and photoelectron spectroscopy. High-resolution mass spectrometry reveals addition products, firmly demonstrating the covalent attachment of the dopant to PCBM molecules. The thermal stability of $\boldsymbol{o}$-AzBnO-DMBI in the blend was tested by annealing thin films of high doping concentration in UHV and by measuring temperaturedependent XPS. We find that the activated dopant renders the doped film more resistant to desorption when approaching the desorption temperature of PCBM in UHV. At room temperature, activation of $\boldsymbol{o}$-AzBnO-DMBI in a bilayer structure was shown to prevent desorption from PCBM under UHV. I-V measurements further prove that UVactivated o-AzBnO-DMBI maintains its doping properties giving rise to conductivities typical for blends of DMBI and PCBM. At high electric field strength, the UV-activated doped PCBM films show no or neglectable hysteresis, but a pronounced hysteresis is observed if o-AzBnO-DMBI is not exposed to UV light. The increased electrical stability can be explained by a suppression of dopant drift due to anchoring of the dopant to PCBM after activation.
The great advantage of our approach is the use of unspecific binding of the intermediately generated reactive dopant toward the host material, offering instant application to a plethora of organic semiconductors. Although chemical derivatization of dopants, either $\mathrm{n}$ - or p-type, may be challenging due to the instabilities resulting from their extreme frontier molecular orbital energies, azide incorporation is possible and has the potential to reduce undesired drift and diffusion in multilayer stacks of OLEDs or OPV and can be applied for $\mathrm{p}-\mathrm{n}$ homojunctions based on polymers. The appropriate chemical derivatization of p-type dopants such as F4TCNQ and F6TCNNQ is ongoing in our laboratories.

\section{EXPERIMENTAL SECTION}

Synthesis. All reactions requiring exclusion of oxygen and moisture were carried out in heat-gun-dried glassware under a dry and oxygen-free nitrogen or argon atmosphere using Schlenk and glovebox techniques. All reagents were obtained from commercial suppliers and were used without further purification if not otherwise stated. Deuterated solvents were purchased from Sigma-Aldrich Laborchemikalien GmbH (Seelze, Germany).

Sample Preparation for Analysis. If not noted otherwise, the dopants and PCBM were dissolved separately in chlorobenzene at a concentration of $5 \mathrm{~g} \mathrm{~L}^{-1}$ for XPS and $10 \mathrm{~g} \mathrm{~L}^{-1}$ for $\mathrm{IR} / \mathrm{I}-V$ measurements and were mixed right before spin-coating. The dopants were kept in solution for 5-10 $\mathrm{min}$, but the PCBM solution was stirred overnight at $50{ }^{\circ} \mathrm{C}$. The films were spin-coated at $1000 \mathrm{rpm}$ for $60 \mathrm{~s}$ in a nitrogen glovebox on a cut $\mathrm{n}$-doped silicon wafer for XPS, intrinsic silicon for IR, and on glass for $I-V$ measurements. Activation by UV light was performed with a GPH135T5L/4 mercury-vapor lamp from Peschl Ultraviolet with a nominal UV-C power of $1.2 \mathrm{~W}$ at $254 \mathrm{~nm}$ in the glovebox. The penlight was mounted on a stand at a distance of $10 \mathrm{~cm}$ above the sample. We measured the UV intensity at the sample position using a Newport power meter 1936-C equipped with a 818 -UV photodiode plus attenuator and obtain a power density of $0.8 \mathrm{~mW} \mathrm{~cm} \mathrm{~cm}^{-2}$ for a $20 \mathrm{~min}$ exposure time.

IR Spectroscopy. For IR transmission measurements, a Fourier transform IR spectrometer (Vertex 80v, Bruker) was used. To prevent absorptions from ambient air $\left(\mathrm{H}_{2} \mathrm{O}\right.$ and $\left.\mathrm{CO}_{2}\right)$, the spectrometer was evacuated to 3 mbar. All samples were recorded near normal transmittance $\left(7^{\circ}\right)$ with an MCT detector and a resolution of $4 \mathrm{~cm}^{-1}$. For each spectrum, 200 scans were averaged.

Electrical Measurements. $I-V$ measurements were performed under a nitrogen atmosphere at room temperature. For this purpose, finger-like structured silver contacts with channel lengths between 25 and $30 \mu \mathrm{m}$ were used. Voltages were applied on these contacts using the $4155 \mathrm{C}$ semiconductor parameter analyzer by Agilent Technologies, which reads out corresponding currents for conductivity determination. Conductivities were then calculated from a set of at least three different samples.

Photoelectron Spectroscopy. Photoelectron spectroscopy (PES) measurements were carried out using a PHI5000 VersaProbe scanning photoelectron spectrometer, which was equipped with a monochromated $\mathrm{Al} \mathrm{K} \alpha$ X-ray source at $1486.7 \mathrm{eV}$ photon energy for X-ray photoelectron spectroscopy (XPS) and a differentially pumped helium discharge lamp operated to achieve $\mathrm{He} \mathrm{I}$ emission at $21.2 \mathrm{eV}$ photon energy for ultraviolet photoelectron spectroscopy (UPS). The focused X-ray spot was approximately $200 \mu \mathrm{m}$ in diameter and was moved to a different position on the sample for longer acquisition in order to avoid radiation damage.

Mass Spectrometry. The mass spectra were recorded using the following instruments: JEOL JMS-700 magnetic sector (EI); Bruker ApexQehybrid 9.4 T FT-ICR (ESI, DART); Finnigan LCQquadrupole ion trap (DART); and Bruker Autoflex Speed (MALDI). 


\section{ASSOCIATED CONTENT}

\section{S Supporting Information}

The Supporting Information is available free of charge on the ACS Publications website at DOI: 10.1021/acs.chemmater.9b01150.

Synthesis, NMR spectroscopy, crystal structure, IR spectroscopy, NMR stability test, UV-vis absorption, TGA/DSC, ESI mass spectra, and XPS analysis (PDF)

\section{AUTHOR INFORMATION}

\section{Corresponding Author}

*E-mail: muellen@mpip-mainz.mpg.de.

ORCID

Lars Müller: 0000-0001-7321-4702

Sebastian Beck: 0000-0003-2194-6842

Robert Lovrincic: 0000-0001-5429-5586

Eric Mankel: 0000-0001-6566-157X

Annemarie Pucci: 0000-0002-9038-4110

Uwe H. F. Bunz: 0000-0002-9369-5387

Klaus Müllen: 0000-0001-6630-8786

\section{Present Address}

${ }^{\dagger}$ University of St Andrews, North Haugh, St Andrews KY16 9SS, United Kingdom.

\section{Author Contributions}

${ }^{*}$ P.R. and F.S.B. contributed equally.

\section{Notes}

The authors declare no competing financial interest.

\section{ACKNOWLEDGMENTS}

We gratefully acknowledge the German Federal Ministry of Education and Research (BMBF) for financial support within the InterPhase project (FKZ 13N13659, 13N13656, $13 \mathrm{~N} 13657$, and 13N13658). We thankfully acknowledge the mass spectroscopy data acquired by the Mass Spectroscopy Facility at OCI Heidelberg under the supervision of Dr. J. Gross. We thank Dr. Frank Rominger for single-crystal X-ray diffraction of our title compounds.

\section{ABBREVIATIONS}

(DMBI) 1,3-dimethyl-2-phenyl-2,3-dihydro-1 $H$ benzoimidazole

(o-MeO-DMBI) 2-(2-methoxyphenyl)-1,3-dimethyl-2,3-dihydro- $1 H$-benzoimidazole

(o-BnO-DMBI) 2-(2-(benzyloxy)phenyl)-1,3-dimethyl2,3-dihydro- $1 H$-benzoimidazole

(o-AzBnO-DMBI) 2-(2-((4-azidobenzyl)oxy)phenyl)-1,3-dimethyl-2,3-dihydro-1H-benzoimidazole

\section{REFERENCES}

(1) Lüssem, B.; Riede, M.; Leo, K. Doping of Organic Semiconductors. Phys. Status Solidi A 2013, 9-43.

(2) Pfeiffer, M.; Leo, K.; Zhou, X.; Huang, J. S.; Hofmann, M.; Werner, A.; Blochwitz-Nimoth, J. Doped Organic Semiconductors: Physics and Application in Light Emitting Diodes. Org. Electron. 2003, 4, 89-103.

(3) Pfeiffer, M.; Fritz, T.; Blochwitz, J.; Nollau, A.; Plönnigs, B.; Beyer, A.; Leo, K. Controlled Doping of Molecular Organic Layers: Physics and Device Prospects. In Advances in Solid State Physics 39; Kramer, B., Ed.; Springer Berlin Heidelberg: Berlin, Heidelberg, 1999; pp 77-90.
(4) Bubnova, O.; Khan, Z. U.; Malti, A.; Braun, S.; Fahlman, M.; Berggren, M.; Crispin, X. Optimization of the Thermoelectric Figure of Merit in the Conducting Polymer Poly(3,4-Ethylenedioxythiophene). Nat. Mater. 2011, 10, 429.

(5) Hynynen, J.; Kiefer, D.; Müller, C. Influence of Crystallinity on the Thermoelectric Power Factor of P3HT Vapour-Doped with F4TCNQ. RSC Adv. 2018, 8, 1593-1599.

(6) Liu, J.; Qiu, L.; Portale, G.; Koopmans, M.; ten Brink, G.; Hummelen, J. C.; Koster, L. J. A. N-Type Organic Thermoelectrics: Improved Power Factor by Tailoring Host-Dopant Miscibility. Adv. Mater. 2017, 29, 1701641.

(7) Shen, Y.; Hosseini, A. R.; Wong, M. H.; Malliaras, G. G. How To Make Ohmic Contacts to Organic Semiconductors. ChemPhysChem 2004, 5, 16-25.

(8) Gross, M.; Müller, D. C.; Nothofer, H.-G.; Scherf, U.; Neher, D.; Bräuchle, C.; Meerholz, K. Improving the Performance of Doped $\pi$ Conjugated Polymers for Use in Organic Light-Emitting Diodes. Nature 2000, 405, 661-665.

(9) Ganzorig, C.; Fujihira, M. Improved Drive Voltages of Organic Electroluminescent Devices with an Efficient P-Type Aromatic Diamine Hole-Injection Layer. Appl. Phys. Lett. 2000, 77, 4211-4213.

(10) D'Andrade, B. W.; Forrest, S. R.; Chwang, A. B. Operational Stability of Electrophosphorescent Devices Containing $\mathrm{p}$ and $\mathrm{n}$ Doped Transport Layers. Appl. Phys. Lett. 2003, 83, 3858-3860.

(11) Arkhipov, V. I.; Emelianova, E. V.; Bässler, H. Quenching of Excitons in Doped Disordered Organic Semiconductors. Phys. Rev. B 2004, 70, 205205.

(12) Arkhipov, V. I.; Emelianova, E. V.; Bässler, H. On the Role of Spectral Diffusion of Excitons in Sensitized Photoconduction in Conjugated Polymers. Chem. Phys. Lett. 2004, 383, 166-170.

(13) Tyagi, P.; Tuli, S.; Srivastava, R. Study of Fluorescence Quenching Due to 2, 3, 5, 6-Tetrafluoro-7, 7', 8, 8'-Tetracyano Quinodimethane and Its Solid State Diffusion Analysis Using Photoluminescence Spectroscopy. J. Chem. Phys. 2015, 142, 054707.

(14) Ferguson, A. J.; Kopidakis, N.; Shaheen, S. E.; Rumbles, G. Quenching of Excitons by Holes in Poly(3-Hexylthiophene) Films. J. Phys. Chem. C 2008, 112, 9865-9871.

(15) Correa-Baena, J. P.; Tress, W.; Domanski, K.; Anaraki, E. H.; Turren-Cruz, S. H.; Roose, B.; Boix, P. P.; Grätzel, M.; Saliba, M.; Abate, A.; et al. Identifying and Suppressing Interfacial Recombination to Achieve High Open-Circuit Voltage in Perovskite Solar Cells. Energy Environ. Sci. 2017, 10, 1207-1212.

(16) Parthasarathy, G.; Shen, C.; Kahn, A.; Forrest, S. R. Lithium Doping of Semiconducting Organic Charge Transport Materials. J. Appl. Phys. 2001, 89, 4986-4992.

(17) Duhm, S.; Salzmann, I.; Bröker, B.; Glowatzki, H.; Johnson, R. L.; Koch, N. Interdiffusion of Molecular Acceptors through Organic Layers to Metal Substrates Mimics Doping-Related Energy Level Shifts. Appl. Phys. Lett. 2009, 95, 093305.

(18) Gao, W.; Kahn, A. Controlledp-doping of Zinc Phthalocyanine by Coevaporation with Tetrafluorotetracyanoquinodimethane: A Direct and Inverse Photoemission Study. Appl. Phys. Lett. 2001, 79, 4040-4042.

(19) Jacobs, I. E.; Aasen, E. W.; Oliveira, J. L.; Fonseca, T. N.; Roehling, J. D.; Li, J.; Zhang, G.; Augustine, M. P.; Mascal, M.; Moulé, A. J. Comparison of Solution-Mixed and Sequentially Processed P3HT:F4TCNQ Films: Effect of Doping-Induced Aggregation on Film Morphology. J. Mater. Chem. C 2016, 4, 3454-3466.

(20) Hynynen, J.; Kiefer, D.; Yu, L.; Kroon, R.; Munir, R.; Amassian, A.; Kemerink, M.; Müller, C. Enhanced Electrical Conductivity of Molecularly P-Doped Poly(3-Hexylthiophene) through Understanding the Correlation with Solid-State Order. Macromolecules 2017, 50, 8140-8148.

(21) Reiser, P.; Müller, L.; Sivanesan, V.; Lovrincic, R.; Barlow, S.; Marder, S. R.; Pucci, A.; Jaegermann, W.; Mankel, E.; Beck, S. Dopant Diffusion in Sequentially Doped Poly(3-Hexylthiophene) Studied by Infrared and Photoelectron Spectroscopy. J. Phys. Chem. C 2018, 122, 14518-14527. 
(22) Müller, L.; Rhim, S.-Y.; Sivanesan, V.; Wang, D.; Hietzschold, S.; Reiser, P.; Mankel, E.; Beck, S.; Barlow, S.; Marder, S. R.; et al. Electric-Field-Controlled Dopant Distribution in Organic Semiconductors. Adv. Mater. 2017, 1701466.

(23) Mor, G. K.; Jones, D.; Le, T. P.; Shang, Z.; Weathers, P. J.; Woltermann, M. K. B.; Vakhshouri, K.; Williams, B. P.; Tohran, S. A.; Saito, T.; et al. Contact Doping with Sub-Monolayers of Strong Polyelectrolytes for Organic Photovoltaics. Adv. Energy Mater. 2014, 4, 1400439.

(24) Dai, A.; Wan, A.; Magee, C.; Zhang, Y.; Barlow, S.; Marder, S. R.; Kahn, A. Investigation of P-Dopant Diffusion in Polymer Films and Bulk Heterojunctions: Stable Spatially-Confined Doping for AllSolution Processed Solar Cells. Org. Electron. 2015, 23, 151-157.

(25) Li, J.; Rochester, C. W.; Jacobs, I. E.; Aasen, E. W.; Friedrich, S.; Stroeve, P.; Moule, A. J. The Effect of Thermal Annealing on Dopant Site Choice in Conjugated Polymers. Org. Electron. 2016, 33, $23-31$.

(26) Ishii, H.; Sugiyama, K.; Ito, E.; Seki, K. Energy Level Alignment and Interfacial Electronic Structures at Organic/Metal and Organic/ Organic Interfaces. Adv. Mater. 1999, 11, 605-625.

(27) Li, J.; Rochester, C. W.; Jacobs, I. E.; Friedrich, S.; Stroeve, P.; Riede, M.; Moulé, A. J. Measurement of Small Molecular Dopant F4TCNQ and $\mathrm{C}_{60} \mathrm{~F}_{36}$ Diffusion in Organic Bilayer Architectures. ACS Appl. Mater. Interfaces 2015, 7, 28420-28428.

(28) Sutton, C.; Risko, C.; Brédas, J.-L. Noncovalent Intermolecular Interactions in Organic Electronic Materials: Implications for the Molecular Packing vs Electronic Properties of Acenes. Chem. Mater. 2016, 28, 3-16.

(29) Li, J.; Koshnick, C.; Diallo, S. O.; Ackling, S.; Huang, D. M.; Jacobs, I. E.; Harrelson, T. F.; Hong, K.; Zhang, G.; Beckett, J.; et al. Quantitative Measurements of the Temperature-Dependent Microscopic and Macroscopic Dynamics of a Molecular Dopant in a Conjugated Polymer. Macromolecules 2017, 50, 5476-5489.

(30) Russ, B.; Robb, M. J.; Brunetti, F. G.; Miller, P. L.; Perry, E. E.; Patel, S. N.; Ho, V.; Chang, W. B.; Urban, J. J.; Chabinyc, M. L.; et al. Power Factor Enhancement in Solution-Processed Organic n-Type Thermoelectrics through Molecular Design. Adv. Mater. 2014, 26, 3473-3477.

(31) Chen, W.; Jiao, W.; Li, D.; Sun, X.; Guo, X.; Lei, M.; Wang, Q.; $\mathrm{Li}, \mathrm{Y}$. Cross Self-n-Doping and Electron Transfer Model in a Stable and Highly Conductive Fullerene Ammonium Iodide: A Promising Cathode Interlayer in Organic Solar Cells. Chem. Mater. 2016, 28, $1227-1235$.

(32) Shlyakhtenko, L. S.; Gall, A. A.; Weimer, J. J.; Hawn, D. D.; Lyubchenko, Y. L. Atomic Force Microscopy Imaging of DNA Covalently Immobilized on a Functionalized Mica Substrate. Biophys. J. 1999, 77, 568-576.

(33) Dhal, P. K.; Babu, G. N.; Sudhakaran, S.; Borkar, P. S. Immobilization of Penicillin Acylase by Covalent Linkage on Vinyl Copolymers Containing Epoxy Groups. Makromol. Chem., Rapid Commun. 1985, 6, 91-95.

(34) Lobnik, A.; Oehme, I.; Murkovic, I.; Wolfbeis, O. S. PH Optical Sensors Based on Sol-Gels: Chemical Doping versus Covalent Immobilization. Anal. Chim. Acta 1998, 367, 159-165.

(35) Freudenberg, J.; Jänsch, D.; Hinkel, F.; Bunz, U. H. F. Immobilization Strategies for Organic Semiconducting Conjugated Polymers. Chem. Rev. 2018, 5598-5689.

(36) Png, R.-Q.; Chia, P.-J.; Tang, J.-C.; Liu, B.; Sivaramakrishnan, S.; Zhou, M.; Khong, S.-H.; Chan, H. S. O.; Burroughes, J. H.; Chua, L.-L.; et al. High-Performance Polymer Semiconducting Heterostructure Devices by Nitrene-Mediated Photocrosslinking of Alkyl Side Chains. Nat. Mater. 2010, 9, 152.

(37) Cho, N.; Yip, H.-L.; Davies, J. A.; Kazarinoff, P. D.; Zeigler, D. F.; Durban, M. M.; Segawa, Y.; O’Malley, K. M.; Luscombe, C. K.; Jen, A. K.-Y. In-Situ Crosslinking and n-Doping of Semiconducting Polymers and Their Application as Efficient Electron-Transporting Materials in Inverted Polymer Solar Cells. Adv. Energy Mater. 2011, 1, $1148-1153$
(38) Bräse, S.; Gil, C.; Knepper, K.; Zimmermann, V. Organic Azides: An Exploding Diversity of a Unique Class of Compounds. Angew. Chem., Int. Ed. 2005, 5188-5240.

(39) Poe, R.; Schnapp, K.; Young, M. J. T.; Grayzar, J.; Platz, M. S. Chemistry and Kinetics of Singlet Pentafluorophenylnitrene. J. Am. Chem. Soc. 1992, 114, 5054-5067.

(40) Marcinek, A.; Platz, M. S.; Chan, S. Y.; Floresca, R.; Rajagopalan, K.; Golinski, M.; Watt, D. Unusually Long Lifetimes of the Singlet Nitrenes Derived from 4-Azido-2,3,5,6-Tetrafluorobenzamides. J. Phys. Chem. 1994, 98, 412-419.

(41) Leyva, E.; Young, M. J. T.; Platz, M. S. High Yields of Formal $\mathrm{CH}$ Insertion Products in the Reactions of Polyfluorinated Aromatic Nitrenes. J. Am. Chem. Soc. 1986, 108, 8307-8309.

(42) Murase, S.; Shibata, K.; Furukawa, H.; Miyashita, Y.; Horie, K. Large Photoinduced Refractive Index Increase in Polymer Films Containing Phenylazide Maintaining Their Transparency and Thermal Stability. Polym. J. 2003, 35, 203-208.

(43) Reiser, A.; Leyshon, L. J.; Johnston, L. Effect of Matrix Rigidity on the Reactions of Aromatic Nitrenes in Polymers. Trans. Faraday Soc. 1971, 67, 2389-2396.

(44) Reddington, S. C.; Rizkallah, P. J.; Watson, P. D.; Pearson, R.; Tippmann, E. M.; Jones, D. D. Different Photochemical Events of a Genetically Encoded Phenyl Azide Define and Modulate GFP Fluorescence. Angew. Chem., Int. Ed. 2013, 52, 5974-5977.

(45) Cai, S. X.; Glenn, D. J.; Kanskar, M.; Wybourne, M. N.; Keana, J. F. W. Development of Highly Efficient Deep-UV and Electron Beam Mediated Cross-Linkers: Synthesis and Photolysis of Bis(Perfluorophenyl) Azides. Chem. Mater. 1994, 6, 1822-1829.

(46) Kim, S. S.; Bae, S.; Jo, W. H. Performance Enhancement of Planar Heterojunction Perovskite Solar Cells by N-Doping of the Electron Transporting Layer. Chem. Commun. 2015, 51, 1741317416.

(47) Naab, B. D.; Zhang, S.; Vandewal, K.; Salleo, A.; Barlow, S.; Marder, S. R.; Bao, Z. Effective Solution- and Vacuum-Processed nDoping by Dimers of Benzimidazoline Radicals. Adv. Mater. 2014, 26, $4268-4272$.

(48) Wei, P.; Oh, J. H.; Dong, G.; Bao, Z. Use of a $1 \mathrm{H}-$ Benzoimidazole Derivative as an n-Type Dopant and to Enable AirStable Solution-Processed n-Channel Organic Thin-Film Transistors. J. Am. Chem. Soc. 2010, 132, 8852-8853.

(49) Kwon, G.; Kim, K.; Choi, B. D.; Roh, J.; Lee, C.; Noh, Y. Y.; Seo, S. Y.; Kim, M. G.; Kim, C. Multifunctional Organic-Semiconductor Interfacial Layers for Solution-Processed Oxide-Semiconductor Thin-Film Transistor. Adv. Mater. 2017, 29, 1607055

(50) Rossbauer, S.; Müller, C.; Anthopoulos, T. D. Comparative Study of the N-Type Doping Efficiency in Solution-Processed Fullerenes and Fullerene Derivatives. Adv. Funct. Mater. 2014, 24, $7116-7124$

(51) Naab, B. D.; Guo, S.; Olthof, S.; Evans, E. G. B.; Wei, P.; Millhauser, G. L.; Kahn, A.; Barlow, S.; Marder, S. R.; Bao, Z. Mechanistic Study on the Solution-Phase n-Doping of 1,3-Dimethyl2-Aryl-2, 3-Dihydro-1H-Benzoimidazole Derivatives. J. Am. Chem. Soc. 2013, 135, 15018-15025.

(52) Lu, M.; Nicolai, H. T.; Wetzelaer, G.-J. A. H.; Blom, P. W. M. N-Type Doping of Poly(p-Phenylene Vinylene) with Air-Stable Dopants. Appl. Phys. Lett. 2011, 99, 173302.

(53) Kiefer, D.; Giovannitti, A.; Sun, H.; Biskup, T.; Hofmann, A.; Koopmans, M.; Cendra, C.; Weber, S.; Anton Koster, L. J.; Olsson, E.; et al. Enhanced N-Doping Efficiency of a Naphthalenediimide-Based Copolymer through Polar Side Chains for Organic Thermoelectrics. ACS Energy Lett. 2018, 3, 278-285.

(54) Zhu, X.-Q.; Zhang, M.-T.; Yu, A.; Wang, C.-H.; Cheng, J.-P. Hydride, Hydrogen Atom, Proton, and Electron Transfer Driving Forces of Various Five-Membered Heterocyclic Organic Hydrides and Their Reaction Intermediates in Acetonitrile. J. Am. Chem. Soc. 2008, 130, 2501-2516.

(55) Naab, B. D.; Himmelberger, S.; Diao, Y.; Vandewal, K.; Wei, P.; Lussem, B.; Salleo, A.; Bao, Z. High Mobility N-Type Transistors 
Based on Solution-Sheared Doped 6,13-Bis(Triisopropylsilylethynyl)-

Pentacene Thin Films. Adv. Mater. 2013, 25, 4663-4667.

(56) Lee, W.-Y.; Wu, H.-C.; Lu, C.; Naab, B.D.; Chen, W.C.; Bao, Z. N-Type Doped Conjugated Polymer for Nonvolatile Memory. Adv. Mater. 2017, 29, 1605166.

(57) Lieber, E.; Rao, C. N. R.; Chao, T. S.; Hoffman, C. W. W. Infrared Spectra of Organic Azides. Anal. Chem. 1957, 29, 916-918.

(58) Yuan, R. D.; Xue, J. D.; Zheng, X. M. Structural Dynamics of Phenyl Azide in Light-Absorbing Excited States: Resonance Raman and Quantum Mechanical Calculation Study. Chin. J. Chem. Phys. 2016, 29, 21-30.

(59) Schwarze, M.; Naab, B. D.; Tietze, M. L.; Scholz, R.; Pahner, P.; Bussolotti, F.; Kera, S.; Kasemann, D.; Bao, Z.; Leo, K. Analyzing the N-Doping Mechanism of an Air-Stable Small-Molecule Precursor. ACS Appl. Mater. Interfaces 2017, 10, 1340-1346.

(60) Gouget-Laemmel, A. C.; Yang, J.; Lodhi, M. A.; Siriwardena, A.; Aureau, D.; Boukherroub, R.; Chazalviel, J. N.; Ozanam, F.; Szunerits, S. Functionalization of Azide-Terminated Silicon Surfaces with Glycans Using Click Chemistry: XPS and FTIR Study. J. Phys. Chem. C 2012, 117, 368-375.

(61) Stenehjem, E. D.; Ziatdinov, V. R.; Stack, T. D. P.; Chidsey, C. E. D. Gas-Phase Azide Functionalization of Carbon. J. Am. Chem. Soc. 2013, 135, 1110-1116.

(62) Chen, F. F.; Wang, F. Electronic Structure of the Azide Group in 3\$-Azido-3\$-deoxythymidine (AZT) Compared to Small Azide Compounds. Molecules 2009, 14, 2656-2668.

(63) Grösser, T.; Prato, M.; Lucchini, V.; Hirsch, A.; Wudl, F. Ringexpansion Des Fullerengerüstes Durch Hochregioselektive Bildung von Diazafulleroiden. Angew. Chem. 1995, 107, 1462-1464.

(64) Cases, M.; Duran, M.; Mestres, J.; Martín, N.; Solà, M. Mechanism of the Addition Reaction of Alkyl Azides to [60]Fullerene and the Subsequent N2extrusion to Form Monoimino-[60]Fullerenes. J. Org. Chem. 2001, 66, 433-442.

(65) Grösser, T.; Prato, M.; Lucchini, V.; Hirsch, A.; Wudl, F. Ring Expansion of the Fullerene Core by Highly Regioselective Formation of Diazafulleroids. Angew. Chem., Int. Ed. Engl. 1995, 34, 1343-1345.

(66) Hirsch, A. Addition Reactions of Buckminsterfullerene (C60). Synthesis 1995, 1995, 895-913.

(67) Nakahodo, T.; Okada, M.; Morita, H.; Yoshimura, T.; Ishitsuka, M. O.; Tsuchiya, T.; Maeda, Y.; Fujihara, H.; Akasaka, T.; Gao, X.; et al. $[2+1]$ Cycloaddition of Nitrene onto C60 Revisited: Interconversion between an Aziridinofullerene and an Azafulleroid. Angew. Chem., Int. Ed. 2008, 47, 1298-1300.

(68) Smith, A. B., III; Tokuyama, H. Nitrene Additions to [60]Fullerene Do Not Generate [6,5] Aziridines. Tetrahedron 1996, $52,5257-5262$.

(69) Knochenmuss, R.; Zenobi, R. MALDI Ionization: The Role of In-Plume Processes. Chem. Rev. 2003, 103, 441-452.

(70) El-Aneed, A.; Cohen, A.; Banoub, J. Mass Spectrometry,Review of the Basics: Electrospray, MALDI, and Commonly Used Mass Analyzers. Appl. Spectrosc. Rev. 2009, 210-230.

(71) Yoo, S. H.; Kum, J. M.; Cho, S. O. Tuning the Electronic Band Structure of Pcbm by Electron Irradiation. Nanoscale Res. Lett. 2011, $6,545-7$.

(72) Irwin, M. D.; Buchholz, D. B.; Hains, A. W.; Chang, R. P. H.; Marks, T. J. P-Type Semiconducting Nickel Oxide as an EfficiencyEnhancing Anode Interfacial Layer in Polymer Bulk-Heterojunction Solar Cells. Proc. Natl. Acad. Sci. 2008, 105, 2783-2787.

(73) Maibach, J.; Mankel, E.; Mayer, T.; Jaegermann, W. The Band Energy Diagram of PCBM-DH6T Bulk Heterojunction Solar Cells: Synchrotron-Induced Photoelectron Spectroscopy on Solution Processed DH6T:PCBM Blends and in Situ Prepared PCBM/ DH6T Interfaces. J. Mater. Chem. C 2013, 1, 7635-7642. 